\title{
Linx
}

Revue des linguistes de l'université Paris X Nanterre

59 | 2008

Les conjonctions en diachronie : parcours sémantiques

\section{Présentation : Les conjonctions en diachronie : parcours sémantiques}

Annie Bertin et Hava Bat-Zeev Shyldkrot

\section{(2) OpenEdition}

\section{Journals}

Édition électronique

URL : http://journals.openedition.org/linx/619

DOI : $10.4000 /$ linx.619

ISSN : 2118-9692

Éditeur

Presses universitaires de Paris Nanterre

Édition imprimée

Date de publication : 1 décembre 2008

Pagination : 7-14

ISSN : 0246-8743

Référence électronique

Annie Bertin et Hava Bat-Zeev Shyldkrot, "Présentation : Les conjonctions en diachronie : parcours sémantiques », Linx [En ligne], 59 | 2008, mis en ligne le 08 juillet 2011, consulté le 21 septembre 2020. URL : http://journals.openedition.org/linx/619; DOI : https://doi.org/10.4000/linx.619 


\title{
Présentation : Les conjonctions en diachronie : parcours sémantiques
}

\author{
Annie Bertin \\ Université Paris Onest Nanterre La Défense - UMR 7114 MoDyCo
}

\section{Hava Bat-Zeev Shyldkrot \\ Université de Tel Aviv}

Ce recueil d'articles résume une partie des travaux faits dans le cadre du Groupe de Recherche «Conjonctions en diachronie ». L'intérêt commun pour l'évolution des conjonctions a incité des chercheurs d'horizons variés à former un groupe de travail autour de ce sujet. Les participants du groupe CD sont affiliés à des institutions françaises et étrangères (Paris 10 - UMR Modyco, Paris IV Sorbonne, Nancy 2 - UMR Atilf, Université de Lyon, Inalco, Université de Potsdam, Université de Sousse, Université de Tel Aviv). Cela fait deux ans que ce groupe se réunit régulièrement plusieurs fois par an à Paris Ouest Nanterre pour explorer différents paramètres relevant des conjonctions. L'objectif du groupe était de traiter, d'un point de vue diachronique, les conjonctions et les locutions conjonctives : formation, déformation, reformation et renouvellement (Meillet, 1912, 1915). S'inscrivant dans différents cadres théoriques (inter alia grammaticalisation, psycho-mécanique de Gustave Guillaume, linguistique intégrale d'Eugène Coseriu, théorie des opérations énonciatives de Culioli 
et HPSG = Head Phrase Structure Grammar), les études visaient, à partir d'une observation détaillée d'exemples attestés dans des corpus constitués, à dégager les systèmes sousjacents à l'évolution de cette classe, en les intégrant dans les débats actuels portant sur le changement linguistique. Cet objectif semble d'autant plus justifié, que les conjonctions forment une classe très problématique qui convoque nombre de difficultés, actuellement au cœur des préoccupations de la linguistique historique et générale. Tout particulièrement, il serait pertinent de comparer les conjonctions aux autres classes d'items dits «connecteurs » ou "subordonnants », tels que les locutions prépositionnelles ou les prépositions, considérées également comme des éléments de « jonction» ou d' «intégration », ou selon d'autres, comme des «mots-outils » servant à assurer la cohésion des unités du discours.

Les conjonctions soulèvent, en effet, une question d'importance capitale pour la linguistique, celle de la catégorisation pertinente. Ce problème de catégorisation semble assez transparent, dans le domaine de la sémantique, où les différentes strates du sens ressortent facilement (Harris 1986, König 1985 inter alia), même si les énoncés se prêtent à des interprétations diverses. Il n'en est pas ainsi dans le domaine syntaxique. Aussi Harris déclare-t-il que non seulement il est difficile de trancher entre une temporelle et une causale, mais qu'il est même souvent peu sûr de distinguer une indépendante d'une subordonnée ou d'une coordonnée. On se trouve donc face à une intrication de chevauchements catégoriels résultant de facteurs variés.

C'est principalement les locutions conjonctives françaises qui ont été au centre des travaux du groupe au départ. La distinction entre la locution conjonctive et la conjonction simple s'est posée immédiatement et a amené à aborder un problème d'ordre général : celui du figement. Ce problème qui, abordé d'un point de vue synchronique, amène à des conclusions univoques, s'avère beaucoup plus complexe quand le point de vue diachronique intervient. L'absence constante de stabilité des conjonctions, tant dans la formation que dans l'emploi et la valeur sémantique, incite à reconsidérer le processus de figement (Gross G.) et plus largement l'influence du dynamisme linguistique. Il s'est avéré que les quelques conjonctions d'origine latine qui se sont maintenues en français manifestent une stabilité syntaxique plus grande que les conjonctions de formation française, tout en accumulant plusieurs sens durant leur évolution ( $c f$. comme comparatif, causal, consécutif). S'intéressant essentiellement aux conjonctions de formation française, on s'est d'abord interrogé sur leur mode de formation, en tenant compte du fait que la base de la conjonction pouvait appartenir à diverses catégories grammaticales : substantif (moment $>$ du moment que), adjectif (tout $>$ tout que), adverbe (bien $>$ bien que), participe passé $(v u>v u$ que), participe présent (pendant $>$ pendant que), préposition (devant $>$ devant que) etc. Par ailleurs, les nombreuses variations de forme, considérées souvent comme pures variantes graphiques (cf ainz que/ains que/eins que), peuvent dissimuler des variantes morphologiques, introduisant des modifications de nature grammaticale ( $c f$. devant ce que, en devant ce que, devant là que). Ces très fréquentes variations dans la formation et l'évolution morphologique ont suscité un intérêt particulier d'autant plus qu'il est difficile de déterminer a priori l'origine de ces variations et leur nature. La question de la disparition et du renouvellement de ces items n'a pas encore été résolue non plus. Il n'est toujours pas clair de savoir pourquoi les conjonctions, contrairement aux autres marqueurs grammaticaux, se renouvellent sans cesse (Meillet 1912). 
Bien que de nombreux travaux aient été consacrés à l'évolution des conjonctions de types variés à partir des années 1980 (Bat Zeev Shyldkrot \& Kemmer (1988), Bertin (1997, 2001), Combettes (1997, 2006a, 2006b), Soutet (1990, 1992), Harris (1986), König (1985, 1988), Kortmann (1997), Marcello-Nizia (2001, 2006), Prévost et Fagard (2007), Vincent (1995) inter alia, certains problèmes d'ordre général concernant cette classe mériteraient toujours une réflexion systématique. Ces recherches lient de différentes manières la forme de la conjonction à son sémantisme. Il apparaît nettement, d'après ces travaux, que les conjonctions temporelles suivent un parcours ordonné (Heine et Kuteva 2002, 2005) et qu'en fonction de la notion temporelle exprimée, il est généralement possible de prévoir dans quel sens la conjonction est susceptible d'évoluer. Pour ne prendre qu'un exemple, très tôt dans les travaux de la grammaticalisation, il a été signalé par Traugott (1980), Bat Zeev Shyldkrot (1987, 1989) que la notion temporelle exprimée par la conjonction pouvait entraîner une évolution sémantique de type différent. Ainsi les conjonctions exprimant la simultanéité comme alors que ou tandis que devenaient assez régulièrement des expressions de concession; de même, une conjonction temporelle exprimant l'antériorité comme puisque devenait très tôt dans la constitution du français, une conjonction à sens causal.

Il n'est donc pas étonnant que les auteurs aient porté une attention particulière à la problématique des divers types de conjonctions temporelles françaises, prises en elles-mêmes ou dans leur rapport avec les conjonctions causales et concessives, rassemblées par certains linguistes (Kortmann 1997) en tant qu'elles expriment un rapport logique. Un article sur le hongrois, langue européenne qui n'appartient pourtant pas à la famille indoeuropéenne, a été incorporé afin de démontrer que parfois les aspects culturels prévalent sur les distinctions de nature typologique et que le parcours d'évolution suivi par les conjonctions peut être similaire dans différentes familles de langues.

Dans son article «La variation que/ce que et la formation des locutions conjonctives en français ", Bernard Combettes place la formation conjonctive au sein de l'évolution syntaxique du français. Il examine l'alternance que/ce que dans le champ des conjonctions temporelles, qui conduit à une double série de locutions avec ou sans le démonstratif ce (par exemple, alors que, dès que vs par ce que, avant ce que); dans l'histoire du français, certaines locutions offrent même deux variantes (après que/après ce que, sans que/sans ce que etc). L'auteur démontre que ces locutions continuent des schémas de formation déjà présents dans le système syntaxique du latin, et que les origines distinctes des deux tours (corrélation de type comparatif dans le cas de que, relation de type relatif dans le cas de ce que) correspondent à des propriétés sémantiques différentes de temps, de mode et d'aspect. Dans ces paires, la conjonction en que constitue le tour non marqué, ce qui explique la disparition de la plupart des conjonctions en ce que.

Hava Bat-Zeev Shyldkrot, dans son article «Réflexions sur l'évolution des conjonctions temporelles en français : le cas de premier que » illustre, en retraçant la genèse d'une locution conjonctive peu étudiée de la langue française, les processus d'évolution des conjonctions temporelles d'antériorité en général. A partir d'un emploi adjectivo-adverbial, la locution premier que émerge dans des constructions qui rappellent au départ le sémantisme initial de primauté de cet item, pour acquérir une valeur temporelle d'antériorité, au point d'en faire un synonyme de avant que. L'auteur soulève 
également le problème de l'abondance des conjonctions temporelles d'antériorité jusqu'en Français Classique, où elles se ramènent toutes à avant que, locution prototypique d'antériorité.

Dans «Sur la genèse des locutions participiales », Thomas Verjans, s'intéresse à une classe de conjonctions relativement délaissée dans les études antérieures sur les conjonctions, telles que pendant que, nonobstant que, durant que. Après avoir recensé les conjonctions formées sur une base participiale (participe passé ou participe présent), puis retracé l'évolution de ces conjonctions, il montre les limites du modèle proposé dans le cadre de la grammaticalisation, et suggère alors de recourir, pour rendre compte de la formation de ce type de conjonctions à un mécanisme alternatif, celui de l'analogie, selon la théorie de Coseriu.

Examinant la diversité des emplois de la locution ainsi que en moyen français, Annie Bertin présente une problématique que l'on retrouve fréquemment dans la distribution de plusieurs locutions conjonctives. Alors qu'en moyen français, cette locution était fort polysémique, présentant les notions comparatives, temporelles, consécutives et constituait un quasi-synonyme de comme, le français, au-delà de l'époque classique, l'utilise uniquement avec la valeur comparative et comme coordonnant. L'auteur se demande dans quelle mesure cette locution relève d'un parcours ordinaire de grammaticalisation ou plutôt d'une grammaticalisation avortée.

Dans son article "Les locutions conjonctives en question(s)", Leila Ben Hamed remet en question la distinction entre conjonction simple et locution conjonctive, terme qui résiste à toute tentative de délimitation, au point de dénier toute légitimité à cette notion. En examinant le statut sémantique et morpho-syntaxique de ces termes, elle conclut qu'il s'agit d'une classe hétérogène, productive et ouverte, ce qui va de pair avec une évolution sémantique constante et avec le processus de la grammaticalisation.

Gerda Hassler étudie "Les conjonctions de causalité et leur grammaticalisation». L'auteur examine la nature du lien causal existant entre les deux propositions reliées par ce type de conjonctions. Puis se basant sur un corpus vaste tiré de Frantext, elle démontre les bases cognitives des locutions conjonctives de causalité et réexamine essentiellement les distinctions entre parce que et puisque d'un point de vue diachronique. Une comparaison entre l'évolution de ces locutions françaises et celle de locutions similaires dans d'autres langues est également présentée.

Olivier Soutet, dans sa contribution «Des concessives extensionnelles aux concessives simples : Contribution à l'étude de la genèse sémantique et historique des locutions conjonctives concessives du français ", traite de l'expression de la concession, à travers les locutions concessives principales en ancien français telles que quoi que, combien que et ja soit que et de manière plus marginale bien que, combien que et, encore plus marginale, encore que. L'auteur examine les soubassements onomasiologiques et sémasiologiques, il conclut alors que la distribution du mécanisme concessif n'est pas arbitraire et dégage des régularités dans la distribution de ce mécanisme.

L'article d'Anna Söres, «A propos de la naissance des conjonctions de subordination en hongrois", traitant d'une langue non indoeuropéenne, présente le grand intérêt de montrer que l'évolution des conjonctions est semblable dans différents types de langues. Elle se base sur le hongrois, où les conjonctions étaient absentes à l'origine, et démontre que, à l'instar de la plupart des langues européennes, 
intitulées Standard Average European (Kortmann 1997), le hongrois a développé une pléthore de conjonctions. Qui plus est, l'auteur présente les étapes de l'évolution de ces conjonctions et démontre, sans surprise, qu'elles suivent le même parcours que celles du français.

Isabelle Weill dans «De tant que à tant que... ne » arrive à démontrer la nonpertinence des définitions des dictionnaires en ce qui concerne les mots grammaticaux. Alors que la locution tant que est examinée et simplement définie, celle de tant que... ne, dont le sens diffère considérablement, n'est guère étudiée. Weill lui attribue un sens essentiellement temporel et considère son évolution comme un fait culturel dû à l'évolution des mentalités.

Bien que l'article de Monia Mokni traite essentiellement d'un adverbe temporel et non d'une conjonction, nous avons cru intéressant de l'incorporer dans ce volume, étant donné qu'il témoigne du même parcours sémantique que les conjonctions traitées. Ainsi il est possible de généraliser et de montrer que même si l'étape syntaxique n'est pas la même, les parcours sémantiques sont similaires, ce qui constitue un argument en faveur de la primauté de la sémantique.

\section{RÉFÉRENCES BIBLIOGRAPHIQUES}

BAT-ZEev SHYLDKROT, H., 1987, "Quand, alors que et tandis que: un cas classique d'évolution sémantique », Romance Notes, 28/1, p. 45-51.

BAT-ZEEV SHYLDKROT, H., 1989, « Conjonctions et expression temporelle-causale en français », Folia Linguistica Historica, X: 1-2, p. 263-281.

BAT-ZEeV ShYldKrot, H., 2005, «Grammaticalisation, changements sémantique et polysémie : le cas de vers et envers», in O. Soutet (éd.), La polysémie, Paris, PUPS, p. 203-229.

BAT-Zeev Shyldkrot, H. \& Kemmer, S., 1988, «Le développement sémantique des conjonctions en français : quelques concepts généraux », Revue Romane, 23/1, p. 9-20.

BERTIN, A., 1997, L'expression de la cause en ancien français, Genève, Droz.

BERTIN, A., 2001, « Maintenant: un cas de grammaticalisation? ", Langue française, 130, p. $42-65$.

CombetTes, B., 1997, «Quelques caractéristiques de l'évolution des "adverbiaux contextuels" », L'Information Grammaticale, 74, p.52-56.

COMBETTES, B., 2006a, «Du niveau textuel au niveau énonciatif dans la grammaticalisation : le rôle du contexte », Langue française, 149, p. 48-60.

COMBETTES, B., 2006b «La formation des locutions conjonctives temporelles : le cas de dès que », in Moline, E., \& al. (éd.), Les connecteurs temporels du français, Amsterdam, Rodopi, p. 1-19. 
COSERIU, E., ([1973] 2007), Sincronía, diacronía e historia. El problema del cambio lingüistico, Madrid, Gredos. [Trad. de T. Verjans Synchronie, diachronie et histoire. Le problème $d u$ changement linguistique, édition Texto!, [en ligne] ].

Gross, G., 1996, Les expressions figées en français, Paris, Ophrys.

Guillaume, G., 1984 (1929), Temps et verbe, théorie des aspects, des modes et des temps, Paris, Champion.

HARRIS, M., 1986, "Aspects of subordination in English and other languages », Bulletin of the John Rylands University Library of Manchester, 69, 1, p.123-126.

Heine, B., \& Kuteva, T., 2002, World Lexicon of grammaticalization, Cambridge, Cambridge University Press.

HeINe, B., \& KuTEVA, T., 2005, Language contact and grammatical change, Cambridge, Cambridge University Press.

KÖNIG, E., 1985, « On the history of concessive connectives in English. Diachronic and synchronic evidence », Lingua, 66, p.1-19.

KÖNIG, E., 1988, "Conditionals, concessive conditionals, and concessives: Areas of contrast, overlap and neutralization » in Traugott, E.C., Meulen, A., Ter, Snitzer Reilly, J., \& Ferguson C., (eds) On conditionals, Cambridge, CUP.

Kortmann, B., 1997, Adverbial Subordination. A Typology and History of Adverbial Subordinators Based on European Languages, Berlin-New York, Mouton de Gruyter.

LEHMANN, C.,1985, «Grammaticalization : Synchronic Variation and Diachronic Change ", Lingua e Stile, XX/3, p. 303-318.

LEHMANN, C., 1982/1985/1995, Thoughts on grammaticalization, Munich, LINCOMEuropa.

MARCHELlO-NiziA, Ch., 2001, "Grammaticalisation et évolution des systèmes grammaticaux », Langue française, 135, p. 33-41.

MARCHELlo-NiziA, Ch., 2006, Grammaticalisation et changement linguistique, Bruxelles, De Boeck.

MeIllet, A., 1912, "L'évolution des formes grammaticales », Scientia (Rivista de scienza), vol XII. [Réimprimé in Linguistique bistorique et linguistique générale, Paris, Champion, 1958].

MeIllet, A., 1915, "Le renouvellement des conjonctions », Annuaire de l'Ecole pratique des Hautes Etudes [Réimprimé in Linguistique bistorique et linguistique générale, Paris, Champion, 1958].

MILLER, Ph. (ed.) 2001, Grammatical interfaces in HPSG, Stanford : CSLI Publications.

Prévost, S., \& FAgARD, B., 2007, «Présentation: grammaticalisation et lexicalisation : la formation d'expressions complexes », Langue française, 156, p. 3-9. 
SAG, I. A., WASOW, Th. \& BENDER, E., 2003, Syntactic Theory : a formal introduction, 2d ed., Chicago, University of Chicago Press.

SOUTET, O., 1990, La concession en français des origines an XVTe siècle, Genève, Droz.

SOUTET, O., 1992, La concession dans les phrases complexes en français, des origines au XV $T^{e}$ siècle, Genève, Droz.

TraugotT, E. C., 1982, «Meaning Change and the Development of Grammatical Markers », Language Sciences, 2/1, p. 44-61.

TRAUGOTT, E.C., 1990, «From less to more situated in language : the unidirectionality of semantic change ", in Adamson, S., and al. (eds.) Papers from the 5th International Conference on English Historical Linguistics, Amsterdam, Benjamins.

VInCENT, N., 1995, «Exaptation and grammaticalization » in Andersen, H. (ed.) Historical Linguistics, Amsterdam/Philadelphia, John Benjamins, p. 433-445. 